\title{
Simultaneous Forward-Backward Prony Estimation
}

\author{
Jim Follum \\ Pacific Northwest National Laboratory \\ james.follum@pnnl.gov \\ Francis Tuffner \\ Pacific Northwest National Laboratory \\ francis.tuffner@pnnl.gov
}

\author{
Md. Arif Khan \\ Schweitzer Engineering Laboratory, Inc. \\ arif_khan@selinc.com \\ Pavel Etingov \\ Pacific Northwest National Laboratory \\ pavel.etingov@pnnl.gov
}

\begin{abstract}
Power system dynamic stability can be evaluated through the analysis of transient oscillations that occur following significant system events. One of the earliest methods for this type of study is Prony analysis, which estimates the system's electromechanical modes. While previous studies have highlighted advantages of performing Prony analysis on data in the forward and backward directions, the proposed method does so simultaneously. As a result, signal poles corresponding to electromechanical modes can be distinguished from spurious poles more reliably. The method also produces a single mode estimate, where independent application in the forward and backward directions would produce two estimates for each mode. The method is validated using simulated and measured power system data.
\end{abstract}

\section{Introduction}

For safe and reliable operation of a power grid, the system's small signal-stability must be maintained. Small-signal stability refers to the system's ability to maintain synchronism after a disturbance [1]. The August 10, 1996 breakup of the Western Electricity Coordinating Council (WECC) system, which resulted in the loss of 30,390 MW of load impacting 7.49 million customers, serves as an example of the catastrophic effects of instability [2].

The severe mismatches between modeled and observed system behavior during the event [2] also highlighted the need for regular system testing and

The Pacific Northwest National Laboratory is operated for the U.S. Department of Energy by Battelle Memorial Institute under Contract DE-AC05-76RL01830. This work was funded by the U.S. DOE through the Grid Modernization Lab Consortium and through Bonneville Power Administration's Technology Innovation program. model validation. In the WECC system, the 1400 MW Chief Joseph dynamic braking resistor is used to excite the system's dynamics during tests [3]. Unplanned system events such as line and generator trips can provide similar excitation. The resulting "ringdown" oscillations can be analyzed for comparison with model-based results and to better understand how system conditions impact stability [4].

Specifically, analysis of ringdown oscillations leads to estimates of the system's dominant inter-area electromechanical modes [1]. The modes dictate the exchange of electrical and mechanical energy between groups of generators in disparate parts of the system. The modes are characterized in part by frequency and damping ratio terms, which are directly related to the frequencies and damping ratios of oscillations observed in measurements of frequency and power. Thus, these oscillations can be analyzed to estimate the electromechanical modes. As long as the damping ratios of all modes are positive, the system is stable. If any of the modal damping ratios become negative, oscillations of power will grow until protective devices operate. This type of line tripping contributed to the cascading event on August 10, 1996 [2]. Thus, knowledge of a system's electromechanical modes is helpful in maintaining stable operation.

The measurements used in such analyses typically come from phasor measurement units (PMUs). They provide GPS-synchronized measurements of voltage phasors, current phasors, and frequency at relatively high reporting rates, typically 30 or 60 frames per second. Time-synchronization allows measurements from across the system to be analyzed together and the high reporting rates allow the oscillations of interest to be captured effectively. In contrast, SCADA measurements are typically not well-suited 
for analyzing oscillations because they are reported asynchronously and relatively infrequently.

With the advent of PMU networks, several algorithms for the analysis of ringdown data were developed $[5,6,7,8]$. The focus of this paper is on an extension of the earliest such algorithm, Prony analysis [9]. Prony analysis is a method of fitting a sum of damped sinusoids to measured data. Several adjustments to the basic algorithm to improve performance at low signal-to-noise ratio (SNR) levels were suggested in a series of papers that are summarized in [10].

One of the challenges of Prony analysis addressed in [10] is distinguishing between the true signal poles, which in the power system application correspond to electromechanical modes, and extraneous poles that are introduced by overfitting the model to account for noise. This problem is also addressed in $[11,12,13]$, but the solution in [10] is most relevant to the present work. The authors of [10] propose analyzing the ringdown in the backward direction so that signal poles appear unstable while extraneous poles remain in the stable region. A closely related approach was recently proposed in [14], which formulates the backward application of Prony in a different though mathematically identical manner and suggests applying Prony in the forward direction as well. The backward Prony approach suggested in [10] was derived for the noiseless case in [15] but holds for moderate amounts of noise. The topic of this paper is an additional modification to Prony analysis that builds upon this earlier work.

The primary contributions of this paper are: 1) the formulation of a constrained nonlinear optimization problem to simultaneously analyze a ringdown in the forward and backward directions and 2) a novel method of distinguishing between signal and extraneous pole estimates. A flowchart describing the overall methodology is provided in Fig. 1. Prony analysis is first performed in the forward and backward directions separately, resulting in two sets of pole estimates.

Next, a method of distinguishing between signal and extraneous pole estimates is applied. The method, which is described in Section 4, considers pole estimates from both the forward and backward applications of Prony, enabling better vetting of signal pole estimates than the methods in [10] and [14], which select signal poles from only one implementation. As a result, each of the system's dominant electromechanical modes is associated with two pole estimates: one from the forward application and one from the backward application. To arrive at a single final estimate for each mode, a constrained nonlinear optimization problem is formulated to simultaneously analyze the

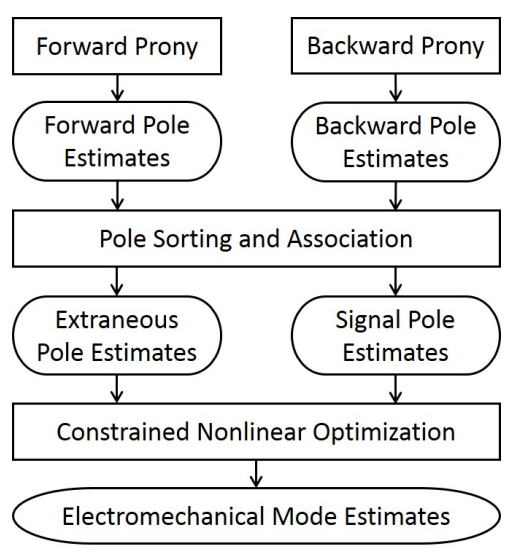

Figure 1. Flowchart describing the overall proposed methodology.

ringdown in the forward and backward directions. This approach is described in Section 3 and provides a modest improvement to estimation accuracy over separate applications of Prony.

Before proposing the simultaneous approach, an overview of the modern Prony algorithm is provided in Section 2. The new algorithms are proposed in Sections 3 and 4, followed by discussion of results from simulated and real-world data in Section 5. Section 6 lists conclusions and expectations for future work.

\section{Overview of Prony Analysis}

Following [9], consider a linear, time-invariant dynamic system brought to initial state $x\left(t_{0}\right)=x_{0}$ by an input. When the input is removed, the system will respond according to the differential equation

$$
\dot{x}=A x .
$$

Here the initiating input, e.g., insertion of the Chief Joseph dynamic brake, is assumed large enough that smaller inputs and disturbances may be ignored. The eigenvalues $\lambda_{i}$, right eigenvectors $p_{i}$, and left eigenvectors $q_{i}$ of the $n \times n$ state matrix $A$ describe the system's dynamics. Of particular interest here are the eigenvalues associated with inter-area electromechanical modes. The associated right eigenvectors define each mode's shape [1]. The solution to (1) can be expressed as [9]

$$
x(t)=\sum_{i=1}^{n} p_{i} q_{i}^{T} x_{0} e^{\lambda_{i} t}
$$

where $t$ denotes time and the transpose operator is denoted with the superscript $T$. 


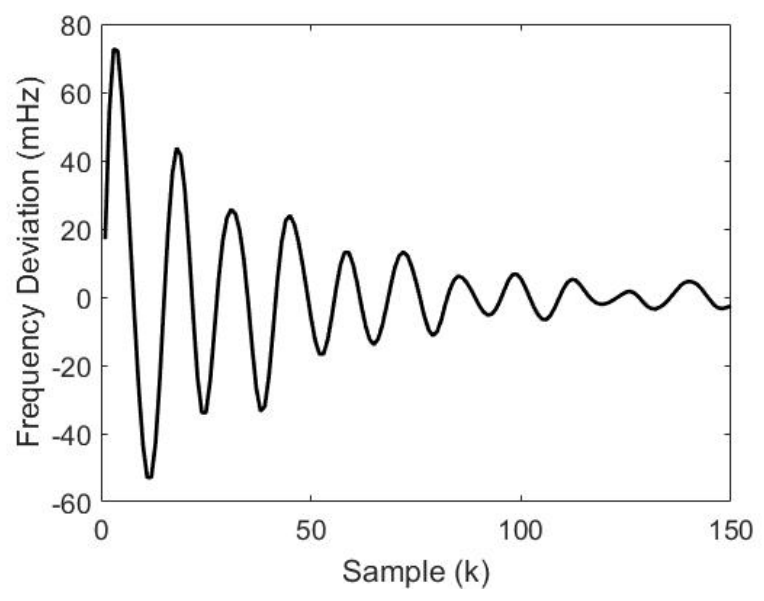

Figure 2. Example of a simulated ringdown after preprocessing.

Now suppose that the $M$ outputs of the system are of the form

$$
y(t)=C x(t),
$$

where $C$ is an $M \times n$ matrix [1]. Inserting (2), the outputs can be expressed as

$$
y(t)=\sum_{i=1}^{n} B_{i} e^{\lambda_{i} t}
$$

where $B_{i}=C p_{i} q_{i}^{T} x_{0}$ is an $M \times 1$ vector containing the output residues for signal pole $\lambda_{i}$. Outputs can be expressed individually as

$$
y_{m}(t)=\sum_{i=1}^{n} B_{m i} e^{\lambda_{i} t}
$$

where $B_{m i}$ denotes the $m^{t h}$ entry of $B_{i}$. When sampled at interval $T$ as the output is measured, (5) becomes

$$
y_{m}(k T)=y_{m}[k]=\sum_{i=1}^{n} B_{m i} z_{i}^{k}, \quad k=0, \ldots, N-1
$$

where $z_{i}=e^{\lambda_{i} T}$ are discrete-time poles. An example of such a signal when all poles are stable is provided in Fig. 2.

The objective of Prony analysis is to fit a measured ringdown $\hat{y}[k]$ to the model given by (6) by selecting the $B_{m i}$ and $z_{i}$. This can be done with the steps that follow. See [16] for a full treatment of the single-channel approach and [17] for a description of the multi-channel version implemented here.
Begin by forming the equation

$$
\underbrace{\left[\begin{array}{c}
y_{1}^{f} \\
y_{2}^{f} \\
\vdots \\
y_{M}^{f}
\end{array}\right]}_{y_{f}}=\underbrace{\left[\begin{array}{c}
Y_{1}^{f} \\
Y_{2}^{f} \\
\vdots \\
Y_{M}^{f}
\end{array}\right]}_{Y_{f}} \underbrace{\left[\begin{array}{c}
a_{1} \\
a_{2} \\
\vdots \\
a_{L}
\end{array}\right]}_{\theta_{f}}
$$

where

$$
\begin{gathered}
y_{m}^{f}=\left[\begin{array}{c}
\hat{y}_{m}[L] \\
\hat{y}_{m}[L+1] \\
\vdots \\
\hat{y}_{m}[N-1]
\end{array}\right] \\
Y_{m}^{f}=\left[\begin{array}{ccc}
\hat{y}_{m}[L-1] & \ldots & \hat{y}_{m}[0] \\
\hat{y}_{m}[L] & \ldots & \hat{y}_{m}[1] \\
\vdots & & \vdots \\
\hat{y}_{m}[N-2] & \ldots & \hat{y}_{m}[N-L-1]
\end{array}\right]
\end{gathered}
$$

and $L$ is the model order. Because only the dominant modes are of interest a reduced order model is constructed, i.e., $L \ll n$. To suppress the effects of noise, $L$ is normally selected to be larger than $n_{\lambda}$, the number of dominant poles in the signal [11]. The well known solution to (7) for the least squares cost function

$$
J\left(\theta_{f}\right)=\left(y_{f}-Y_{f} \theta_{f}\right)^{T}\left(y_{f}-Y_{f} \theta_{f}\right)
$$

is given by

$$
\hat{\theta}_{f}=\left(Y_{f}^{T} Y_{f}\right)^{-1} Y_{f}^{T} y_{f}=Y_{f}^{\dagger} y_{f}
$$

where $\dagger$ denotes the pseudoinverse. In practice, it is advantageous to calculate the pseudoinverse using singular value decomposition (SVD) to alleviate ill-conditioning [10]. MATLAB's pinv(.) function calculates the pseudoinverse in this manner by default.

Using the estimate given by (11) the $z_{i}$ can be estimated as the roots of the characteristic equation

$$
z^{L}-\left(\hat{a}_{1} z^{L-1}+\hat{a}_{2} z^{L-2}+\cdots+\hat{a}_{L} z^{0}\right)=0 .
$$

The complex roots of this equation appear in conjugate pairs. They are stable if their magnitudes are less than one and unstable if their magnitudes are greater than one (see [18] or other texts on the fundamentals of systems). For moderate SNR levels, $n_{\lambda}$ pole estimates will correspond to signal poles, leaving $L-$ $n_{\lambda}$ extraneous pole estimates. Extraneous poles, used to account for noise, tend to have magnitudes less than one for moderate noise cases [15]. See Fig. 3, which indicates the results of standard (forward) Prony 


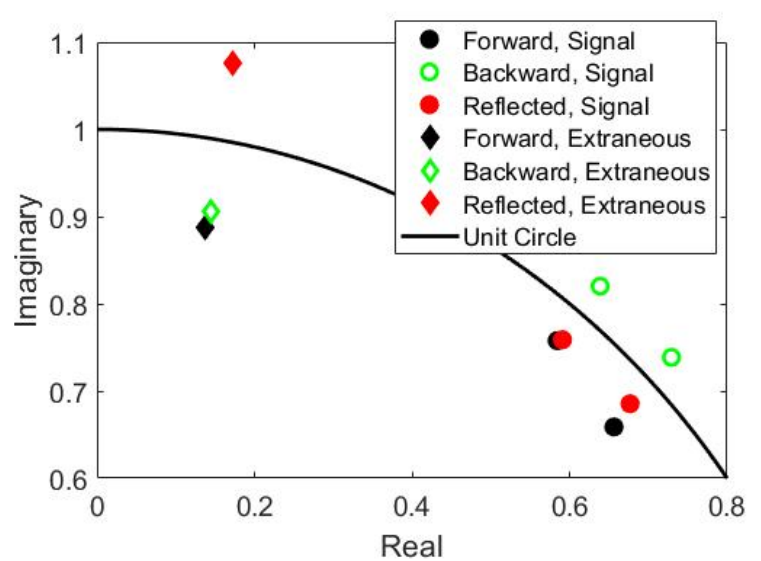

Figure 3. Example signal and extraneous discrete-time pole locations from forward and backward application of Prony. The example was generated using simulation data from a model with known modes.

analysis in black: two signal poles and one extraneous pole, all within the unit circle. Distinguishing between signal and extraneous poles is addressed in Section 4. The continuous-time mode estimates corresponding to the roots of (12) are given by

$$
\hat{\lambda}_{i}=\ln \left(\hat{z}_{i}\right) / T
$$

with associated frequency and damping ratio given by [1]

$$
\hat{f}_{i}=\frac{\operatorname{imag}\left(\hat{\lambda}_{i}\right)}{2 \pi}
$$

and

$$
\hat{\zeta}_{i}=-\cos \left(\angle \hat{\lambda}_{i}\right)
$$

With the poles estimated, a reduced-order version of (6) is used to estimate the output residues. Expanding (6) for the $L$ pole estimates leads to

$$
\underbrace{\left[\begin{array}{c}
\hat{y}_{m}[0] \\
\hat{y}_{m}[1] \\
\vdots \\
\hat{y}_{m}[N-1]
\end{array}\right]}_{\tilde{y}_{m}}=\underbrace{\left[\begin{array}{cccc}
\hat{z}_{1}^{0} & \hat{z}_{2}^{0} & \cdots & \hat{z}_{L}^{0} \\
\hat{z}_{1}^{1} & \hat{z}_{2}^{1} & \cdots & \hat{z}_{L}^{1} \\
\vdots & \vdots & \ddots & \vdots \\
\hat{z}_{1}^{N-1} & \hat{z}_{2}^{N-1} & \ldots & \hat{z}_{L}^{N-1}
\end{array}\right]}_{Z} \underbrace{\left[\begin{array}{c}
B_{m 1} \\
B_{m 2} \\
\vdots \\
B_{m L}
\end{array}\right]}_{B_{m}}
$$

with solution

$$
\hat{B}_{m}=Z^{\dagger} \tilde{y}_{m}
$$

With all parameters estimated, the modeled signal can be constructed by inserting parameter values into (6). Because modes are represented by a complex

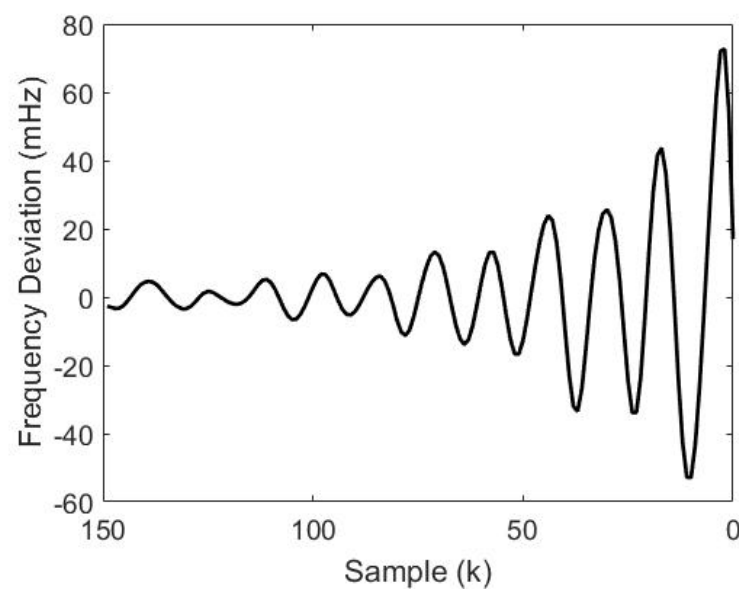

Figure 4. Example signal from Fig. 2 flipped for analysis with a backward application of Prony.

conjugate pair of poles, the contribution of mode $i$ to signal $m$ can be constructed as

$$
y_{m i}[k]=2 \times \operatorname{Real}\left\{\hat{B}_{m i} \hat{z}_{i}^{k}\right\} .
$$

A metric that is often used to rank the contributions of individual poles is the pseudo energy given by [13]

$$
E_{i}=\sum_{m=1}^{M}\left[\sum_{k=0}^{N-1} y_{m i}^{2}[k]\right]
$$

\section{Simultaneous Forward-Backward Prony Estimation}

The proposed method begins with the backward Prony approach of [10]. Flipping the signal so that sample $N-1$ comes first (see Fig. 4) leads to the rearrangement of (7)-(9) as

$$
\underbrace{\left[\begin{array}{c}
y_{1}^{b} \\
y_{2}^{b} \\
\vdots \\
y_{M}^{b}
\end{array}\right]}_{y_{b}}=\underbrace{\left[\begin{array}{c}
Y_{1}^{b} \\
Y_{2}^{b} \\
\vdots \\
Y_{M}^{b}
\end{array}\right]}_{Y_{b}} \underbrace{\left[\begin{array}{c}
b_{1} \\
b_{2} \\
\vdots \\
b_{L}
\end{array}\right]}_{\theta_{b}}
$$

where

$$
y_{m}^{b}=\left[\begin{array}{c}
\hat{y}_{m}[N-L-1] \\
\hat{y}_{m}[N-L-2] \\
\vdots \\
\hat{y}_{m}[0]
\end{array}\right]
$$


and

$$
Y_{m}^{b}=\left[\begin{array}{ccc}
\hat{y}_{m}[N-L] & \ldots & \hat{y}_{m}[N-1] \\
\hat{y}_{m}[N-L-1] & \ldots & \hat{y}_{m}[N-2] \\
\vdots & & \vdots \\
\hat{y}_{m}[1] & \ldots & \hat{y}_{m}[L]
\end{array}\right] .
$$

The least squares cost function and solution follow as, respectively,

$$
J\left(\theta_{b}\right)=\left(y_{b}-Y_{b} \theta_{b}\right)^{T}\left(y_{b}-Y_{b} \theta_{b}\right)
$$

and

$$
\hat{\theta}_{b}=Y_{b}^{\dagger} y_{b} .
$$

Denote the roots of the characteristic equation

$$
u^{L}-\left(\hat{b}_{1} u^{L-1}+\hat{b}_{2} u^{L-2}+\cdots+\hat{b}_{L} u^{0}\right)=0
$$

as $\hat{u}_{i}$. As with the forward application of Prony to signals with moderate noise, $n_{\lambda}$ of these pole estimates will correspond to dominant electromechanical modes. Frequency, damping, shape, and pseudo-energy can be calculated with obvious analogs to (13)-(19). Because the signal is being analyzed in the backward direction, the signal poles corresponding to stable modes will appear unstable with magnitudes greater than one. Because extraneous poles account for noise, they continue to appear within the unit circle [15]. See Fig. 3, which indicates the results of backward Prony analysis in green: two signal poles outside the unit circle and one extraneous pole within the unit circle.

Now consider the pole estimates

$$
\hat{v}_{i}=\frac{1}{\hat{u}_{i}^{*}},
$$

which have been reflected across the unit circle. Here * denotes the complex conjugate. As displayed in Fig. 3 , reflected signal poles fall within the unit circle near pole estimates from the forward application of Prony. The reflected extraneous signal poles fall outside of the unit circle, making it possible to establish an initial distinction between signal and extraneous poles. Further discussion and a method for associating reflected signal pole estimates with pole estimates from the forward application of Prony are provided in Section 4.

Using the algorithm to be described in Section 4, assume a subset of the forward and reflected pole estimates have been categorized into $n_{\lambda}$ pairs of signal poles such that

$$
\hat{z}_{j}^{\prime} \approx \hat{v}_{j}^{\prime} \quad, \quad j=1,2, \ldots, n_{\lambda} .
$$

Here the ' denotes that the pole estimate has been classified as a signal pole. With two estimates for each system mode, there is a difficulty in determining which frequency and damping ratio values to report. Analyzing signals separately led to a similar challenge that was addressed with a multi-channel algorithm in [17]. The objective of the proposed method is to reduce these pairs of estimates to single values.

To do so, consider the constrained nonlinear optimization problem

$$
\min _{\theta_{f}, \theta_{b}}\left[J\left(\theta_{f}\right)+J\left(\theta_{b}\right)\right]
$$

such that

$$
\begin{gathered}
\hat{z}_{j}^{\prime \prime}=\hat{v}_{j}^{\prime \prime}, \quad j=1, \ldots, n_{\lambda} \\
\left|\hat{f}_{j}^{\prime \prime}-\hat{f}_{j}^{\prime}\right|<\Delta f, \quad j=1, \ldots, n_{\lambda} \\
\left|\hat{\zeta}_{j}^{\prime \prime}-\hat{\zeta}_{j}^{\prime}\right|<\Delta \zeta, \quad j=1, \ldots, n_{\lambda} \\
\hat{z}_{j}^{\ddagger \ddagger}-\hat{z}_{j}^{\ddagger}=0, \quad j=n_{\lambda}+1, \ldots, L \\
\hat{v}_{j}^{\ddagger \ddagger}-\hat{v}_{j}^{\ddagger}=0, \quad j=n_{\lambda}+1, \ldots, L
\end{gathered}
$$

The cost function minimized in (28) combines the cost functions from the forward and backward applications of Prony given by (10) and (23), respectively. Essentially, a set of mode estimates is sought that provide a good fit to the measurements when analyzed in both the forward and backward directions.

In (29) - (31), " denotes signal pole estimates produced by solving the optimization problem. Constraint (29) ensures that the signal pole estimates obtained by rooting $\theta_{f}$ and $\theta_{b}$ are identical, leading to one signal pole estimate for each pair in (27). For results in this paper, initial values for the optimization algorithm were selected as

$$
\hat{z}_{j}^{\prime \prime}=\frac{\left(\hat{z}_{j}^{\prime}+\hat{v}_{j}^{\prime}\right)}{2} \quad, \quad j=1,2, \ldots, n_{\lambda} .
$$

Constraints (30) and (31) ensure that pole estimates remain associated with the same electromechanical mode by preventing frequency and damping ratio estimates from changing dramatically.

Constraints (32) and (33) dictate that final extraneous pole estimates, denoted with $\ddagger$, remain unchanged from their initial values, which are denoted with $\ddagger$. Thus, the extraneous pole estimates are not allowed to influence the selection of signal pole estimates.

MATLAB's $f$ mincon function was used to solve the constrained optimization problem to generate the results presented in this paper. This function allows various optimization algorithms to be used, but results were 


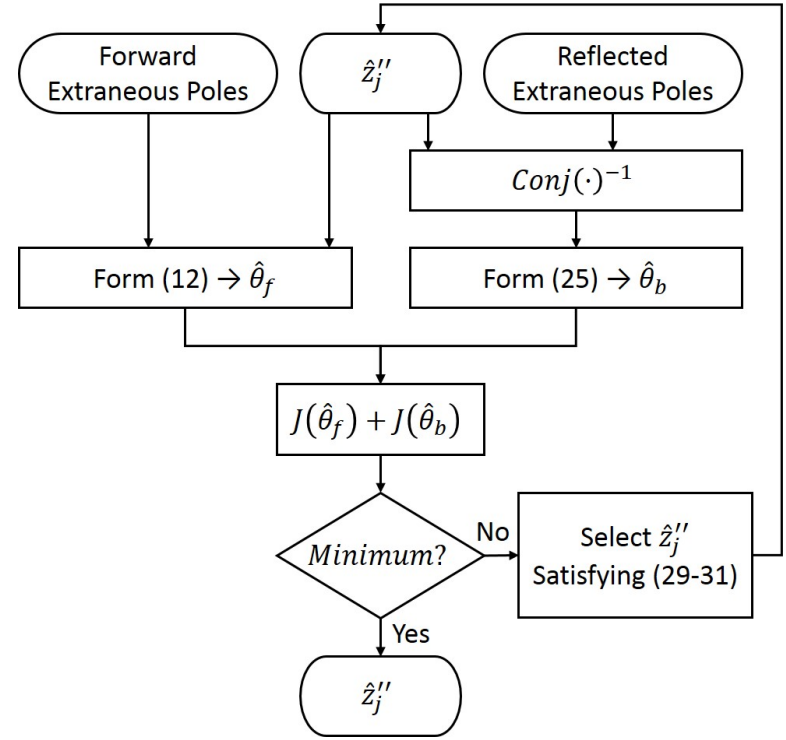

Figure 5. Flowchart describing the iterative process used to solve the optimization problem.

consistent for the several algorithms that were tested. For final results, the default interior-point algorithm was utilized.

A generic flowchart describing the iterative process is presented in Fig. 5. At each iteration, the extraneous pole estimates, which are held constant, and signal pole estimates, which are updated at each iteration, are used to form the characteristic equations in (12) and (25). The MATLAB function poly is then used to find the $a_{i}$ and $b_{i}$ coefficients of the characteristic equations. The cost functions (10) and (23) can then be calculated and combined. If the optimization algorithm determines that the minimum has been achieved, iteration ceases and the final signal pole estimates are returned.

\section{Associating Signal Poles}

In the previous section, it was assumed that a subset of the forward and reflected pole estimates had been categorized into pairs of signal pole estimates. Each pair was then used to initialize the optimization algorithm. A novel method of performing this categorization is proposed in this section to automate the process.

To begin, consider the following list of assumptions about forward and reflected pole estimates established in $[10,15]$ :

a) Stable signal poles are located within the unit circle

b) Unstable signal poles are located outside of the unit circle c) For forward pole estimates, all extraneous poles are located within the unit circle

d) For reflected pole estimates, all extraneous poles are located outside of the unit circle

These assumptions hold in the noiseless case [15] and tend to hold for moderate amounts of noise [10]. To mitigate the risk of these assumptions failing, the algorithm proposed here also requires forward and reflected signal pole estimates to be closely spaced, as in Fig. 3. The initial signal poles in (34) are calculated based on these associated poles.

Based on the listed assumptions, unstable signal poles are readily identified with a forward application of Prony as those outside the unit circle because all other pole estimates are located inside the unit circle. Similarly, stable signal poles are readily identified with a backward application of Prony as those within the unit circle because all other pole estimates are located outside of the unit circle. Thus, the association algorithm is divided into two phases. In the first phase, stable signal poles are identified based primarily on the reflected pole estimates. This phase proceeds as follows:

1. Collect all pole estimates within the unit circle

2. Sort reflected pole estimates according to pseudo-energy, beginning with the most energetic

3. Find the forward pole estimate closest to the currently considered reflected pole estimate

4. Check if the poles are within a frequency and damping ratio tolerance of each other

- If so, associate the poles with each other as signal poles and remove the forward pole estimate from further consideration as a pair

- If not, classify the reflected pole estimate as an extraneous pole

5. Repeat steps 3-4 for remaining reflected pole estimates

Each step can be carried out objectively, allowing the process to be automated to obtain the results presented in this paper.

The second phase of the algorithm identifies unstable signal poles based primarily on the results of the forward application of Prony. The steps of the first phase repeat for pole estimates outside the unit circle, replacing reflected with forward and vice versa. Practically, most analyzed signals will not contain unstable poles, so phase two is often unnecessary. Note, though, that the forward application of Prony is still used to identify stable signal poles in phase one and in the simultaneous 
solution described in Section 3. In previously published methods, e.g., [10, 15, 14], the forward pole estimates are discarded when the system is stable.

Extraneous poles that violate assumptions $a$ or $b$ will tend to have low pseudo-energy [13] and will thus be considered last, by which point nearby signal poles will already be associated with each other. In this way, the algorithm provides robustness against falsely identifying an extraneous pole as a signal pole. Additional criteria can also be used to remove estimates from consideration, e.g., setting a threshold for minimum pseudo-energy. For the results reported in this paper, only mode estimates with frequencies less than $1 \mathrm{~Hz}$ were considered because this is the approximate range of inter-area electromechanical modes. Other types of oscillation modes may be present in the analyzed signal at higher frequencies, but they are considered as "noise" modes for this application. The frequency and damping ratio tolerances referenced in step 4 above were set to $0.05 \mathrm{~Hz}$ and $10 \%$, respectively. Based on extensive testing, the algorithm was found to be relatively insensitive to these parameters.

\section{Method Validation}

To demonstrate the utility of the algorithms proposed in Sections 3 and 4, they were tested with simulated and measured data. Simulation results are useful because the true mode values are known from the model, so statistical performance can be evaluated. Successful analysis of measured data demonstrates that the methods have practical value in real-world applications.

All data was processed in a similar manner. First, linear combinations of voltage angle data were formed to suppress common signal components and emphasize electromechanical oscillations between areas. Next, a first-order derivative filter was applied to obtain estimates of frequency deviation about the nominal system frequency. The initial sampling rates of the simulated and measured data were 30 and 60 samples per second, respectively. To obtain data that more narrowly covered the frequency range of the inter-area dynamics of the systems, the data was passed through a Parks-McClellan finite impulse response (FIR) anti-aliasing filter and downsampled to five samples per second. The filter orders were 72 for data at 30 samples per second and 145 for data at 60 samples per second. A first-order high-pass infinite impulse response (IIR) Butterworth filter was then applied to remove low-frequency trends. Similar preprocessing approaches are described in $[19,20]$.

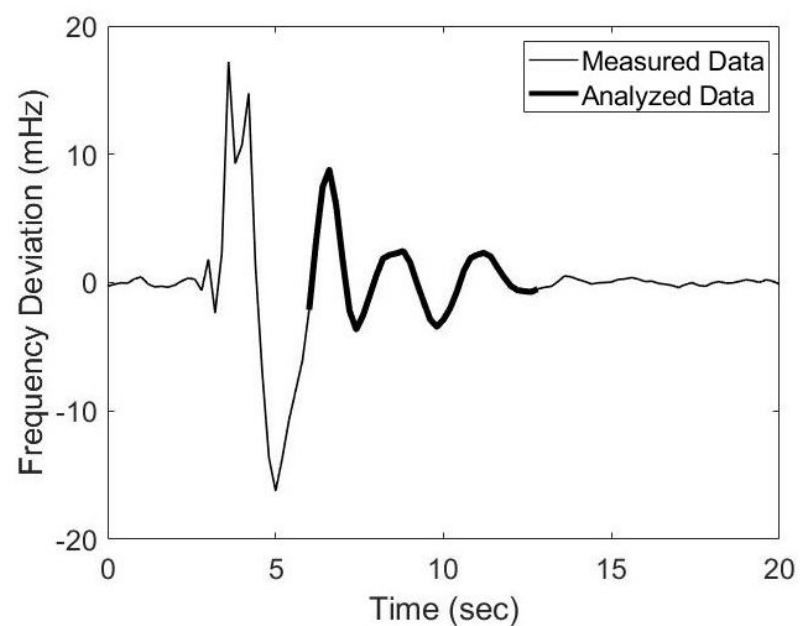

Figure 6. Measured ringdown used to demonstrate the algorithms. The portion included in the analysis is bold.

\subsection{Results from Measured Data}

The methods were evaluated by applying them to ringdowns following unplanned generator trips. To demonstrate the algorithm's operation, one such event will be discussed in detail. The event occurred in the WECC system in April 2018. Further details are withheld to maintain confidentiality. A plot of the analyzed data is provided in Fig. 6. Twenty minutes of ambient data following the ringdown was also analyzed with the Yule-Walker algorithm [21, 22]. Agreement between ambient and ringdown methods, which operate very differently, provides validation for the analysis when applied to adjacent datasets, where the electromechanical modes are expected to be very similar.

Results are presented in Table 1. The forward application of Prony resulted in a spurious estimate at $0.2606 \mathrm{~Hz}$, while the backward application resulted in spurious estimates at $0.2672 \mathrm{~Hz}$ and $0.8174 \mathrm{~Hz}$. If only one application were considered, it could be difficult to tell whether these estimates are valid. In this case, the algorithm described in Section 4 removed them from consideration when the simultaneous algorithm was employed. As a result, only true inter-area modes are reflected in the estimates from the simultaneous Prony algorithm. Both of these mode frequencies are commonly observed in the WECC system. The results are further validated by the Yule-Walker estimate for the mode near $0.41 \mathrm{~Hz}$. The $0.79 \mathrm{~Hz}$ mode was too weak to be observed in ambient data. 
Table 1. Mode Estimates from Measured Data.

\begin{tabular}{|cc|cc|cc|cc|}
\hline \multicolumn{2}{|c|}{ Forward } & \multicolumn{2}{|c|}{ Backward } & \multicolumn{2}{c|}{ Simultaneous } & \multicolumn{2}{c|}{ Yule-Walker } \\
\hline $\mathrm{f}(\mathrm{Hz})$ & $\zeta(\%)$ & $\mathrm{f}(\mathrm{Hz})$ & $\zeta(\%)$ & $\mathrm{f}(\mathrm{Hz})$ & $\zeta(\%)$ & $\mathrm{f}(\mathrm{Hz})$ & $\zeta(\%)$ \\
\hline \hline 0.4350 & 9.4452 & 0.4112 & 12.6540 & 0.4138 & 12.3475 & 0.4082 & 12.6050 \\
\hline 0.7859 & 19.9598 & 0.7932 & 15.3760 & 0.7941 & 15.1572 & & \\
\hline 0.2606 & -0.3737 & 0.2672 & 25.9220 & & & & \\
\hline \multicolumn{1}{|c|}{} & 0.8174 & -16.7164 & & & & \\
\hline
\end{tabular}

\subsection{Results from Simulated Data}

Simulated data was generated using the MinniWECC model, a complete description of which is presented in [23] and the references therein. The MinniWECC is a simplified model of the WECC system obtained by equivalencing generation for many areas into single generators and including only transmission lines with significant length and a rating of at least $230 \mathrm{kV}$. In all, the model has 34 generators, 115 lines and high-voltage transformers, 54 generator and load transformers, 19 load buses, and 2 DC lines. For data generation, the nonlinear model is linearized about an operating point and represented in state-space form.

The model also includes a representation of the 1400 MW dynamic braking resistor used in real-world system tests [3]. To generate data, the brake was inserted for 0.5 seconds to reflect real-world testing procedures. The resulting ringdown oscillations were analyzed to estimate the system's dominant electromechanical modes, which are listed in Table 2 for the two simulation cases examined in this section. The cases differ in the damping ratios of modes 1 and 2. For each case, 500 trials were generated, each with a unique realization of the load noise driving the system and the measurement noise added to the model's output. Calculating the root mean squared error (RMSE) of the estimates over these trials provides an indication of each algorithm's statistical performance. This approach is known as a Monte Carlo simulation [24]. Examples of the three analyzed signals from simulation case 2 after preprocessing are presented in Fig. 7. A model order of 30 was selected by evaluating performance for a variety of model orders. Similar results can be obtained for model orders in the neighborhood of 30 .

Results from the Monte Carlo trials are presented in Figs. 8 and 9 and Tables 3 and 4. Unsurprisingly, performance degrades for modes 1 and 2 in simulation case 2 due to the increased damping ratios of the modes. Tables 3 and 4 reflect characteristic behavior of the proposed method, namely, that its estimates tend to lie between the forward and backward estimates, close
Table 2. Frequency and damping ratio of the dominant electromechanical modes in the simulation cases.

\begin{tabular}{|c|cc|cc|}
\hline & \multicolumn{2}{|c|}{ Simulation Case 1 } & \multicolumn{2}{c|}{ Simulation Case 2 } \\
\hline Mode & $\mathrm{f}(\mathrm{Hz})$ & $\zeta(\%)$ & $\mathrm{f}(\mathrm{Hz})$ & $\zeta(\%)$ \\
\hline \hline 1 & 0.22 & 7.0 & 0.22 & 10.0 \\
\hline 2 & 0.37 & 5.0 & 0.37 & 12.0 \\
\hline 3 & 0.62 & 6.2 & 0.62 & 6.2 \\
\hline 4 & 0.73 & 6.6 & 0.73 & 6.6 \\
\hline
\end{tabular}

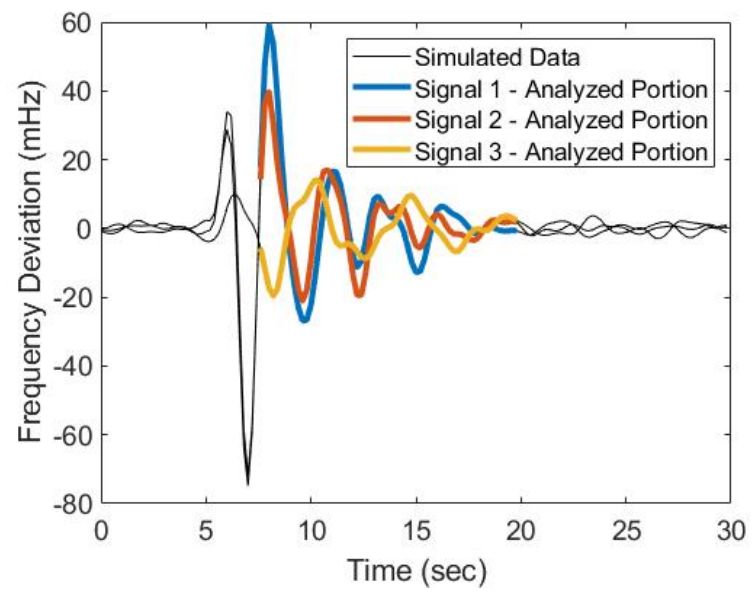

Figure 7. Example ringdowns from simulation case 2. The portions of the ringdowns included in the analyses are indicated with bold lines. 
Table 3. RMSE of frequency and damping ratio estimates from simulation case 1.

\begin{tabular}{|c|ccc|ccc|}
\hline & \multicolumn{3}{|c|}{ Frequency RMSE $(\mathrm{mHz})$} & \multicolumn{3}{c|}{ Damping RMSE (\%) } \\
\hline Mode & Forward & Backward & Simultaneous & Forward & Backward & Simultaneous \\
\hline \hline 1 & 4.3 & 3.3 & 3.6 & 1.9 & 1.7 & 1.6 \\
\hline 2 & 0.9 & 0.8 & 0.7 & 0.2 & 0.2 & 0.2 \\
\hline 3 & 6.2 & 8.8 & 7.4 & 0.9 & 1.0 & 0.9 \\
\hline 4 & 4.6 & 3.6 & 3.1 & 1.1 & 1.8 & 1.5 \\
\hline
\end{tabular}
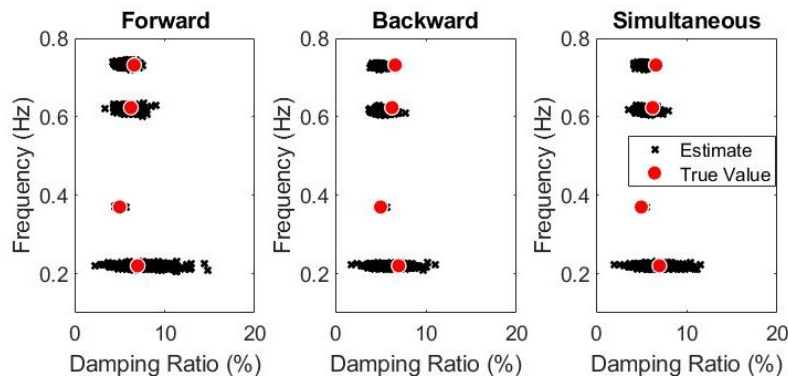

Figure 8. Mode estimation results for simulation case 1. Each estimate is indicated with a black $x$, and true values are indicated with a red dot.
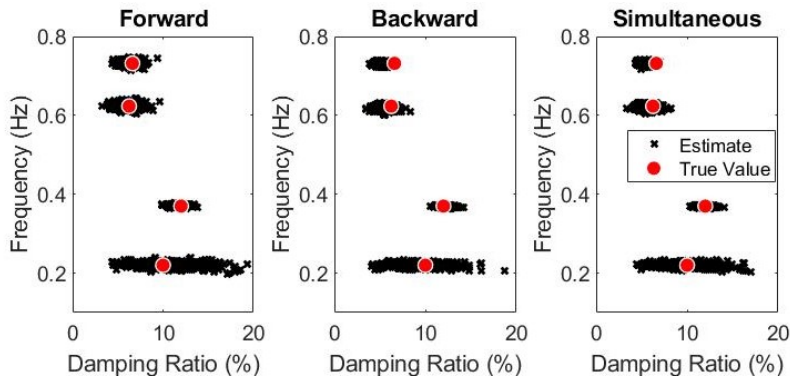

Figure 9. Mode estimation results for simulation case 2 . Each estimate is indicated with a black $x$, and true values are indicated with a red dot.

to the better of the two. Rather than a significant improvement in accuracy, the proposed methods are advantageous because they reliably identify which pole estimates correspond to electromechanical modes and produce a single estimate for each one.

The compromise between the forward and backward applications of Prony is also apparent in the cost function values of Table 5. When Prony is applied in the forward and backward directions separately, their combined cost function is slightly lower than when they are applied simultaneously. The simultaneous application requires that the mode estimates be identical in the forward and backward direction. This additional constraint causes the slight increase in the cost function.
The simulation results also demonstrated that the proposed method can be used practically. The optimization algorithm never failed to converge across the 1000 trials. On average, the optimization ran in 24 milliseconds on a desktop computer. The required runs of Prony analysis in the forward and backward directions required a combined average of under 2 milliseconds. Though the optimization requires more computational burden than the classic Prony algorithm, 24 milliseconds is not a significant burden, especially considering that Prony analysis is typically used in the offline environment.

\section{Conclusion}

In this paper, a novel method for analyzing PMU measurements containing ringdown oscillations is proposed. Estimates of electromechanical modes, which determine the power system's small signal stability, are obtained by fitting a model to the measurements in the forward and backward direction simultaneously. This approach allows estimates corresponding to electromechanical modes to be distinguished from extraneous poles reliably. Where analyzing the signal in the forward and backward directions independently would produce two estimates for each mode, the simultaneous solution produces a single estimate. The proposed solution was shown to offer comparable estimate accuracy to the conventional Prony methods using simulation studies. It's practical utility was also verified using actual system measurements.

\section{References}

[1] P. Kundur, Power System Stability and Control. New York: McGraw-Hill, Inc., 1994.

[2] D. N. Kosterev, C. W. Taylor, and W. A. Mittelstadt, "Model validation for the August 10, 1996 WSCC system outage," Power Systems, IEEE Transactions on, vol. 14, no. 3, pp. 967-979, 1999.

[3] J. F. Hauer, W. A. Mittelstadt, K. E. Martin, J. W. Burns, H. Lee, J. W. Pierre, and D. J. Trudnowski, "Use of the WECC WAMS in wide-area probing tests for validation of system performance and modeling," Power Systems, IEEE Transactions on, vol. 24, no. 1, pp. 250-257, 2009. 
Table 4. RMSE of frequency and damping ratio estimates from simulation case 2.

\begin{tabular}{|c|ccc|ccc|}
\hline & \multicolumn{3}{|c|}{ Frequency RMSE $(\mathrm{mHz})$} & \multicolumn{3}{c|}{ Damping RMSE (\%) } \\
\hline Mode & Forward & Backward & Simultaneous & Forward & Backward & Simultaneous \\
\hline \hline 1 & 6.5 & 4.9 & 5.3 & 3.1 & 2.3 & 2.4 \\
\hline 2 & 2.7 & 2.3 & 2.1 & 0.6 & 0.6 & 0.5 \\
\hline 3 & 6.1 & 6.6 & 5.4 & 0.9 & 0.9 & 0.9 \\
\hline 4 & 5.0 & 3.4 & 3.6 & 0.9 & 1.5 & 1.2 \\
\hline
\end{tabular}

Table 5. Average cost function values.

\begin{tabular}{|c|cc|}
\hline Case & $\begin{array}{c}\text { Forward + Backward } \\
J\left(\theta_{f}\right)+J\left(\theta_{b}\right)\end{array}$ & $\begin{array}{c}\text { Simultaneous } \\
\left(\theta_{f}, \theta_{b}\right)\end{array}$ \\
\hline \hline 1 & $759 \mathrm{e}-9$ & $769 \mathrm{e}-9$ \\
\hline 2 & $266 \mathrm{e}-9$ & $280 \mathrm{e}-9$ \\
\hline
\end{tabular}

[4] Z. Hou, J. Follum, P. Etingov, F. Tuffner, D. Kosterev, and G. Matthews, "Machine learning of factors influencing damping and frequency of dominant inter-area modes in the WECC interconnect," in 2018 IEEE International Conference on Probabilistic Methods Applied to Power Systems (PMAPS), pp. 1-6, June 2018.

[5] I. Kamwa, R. Grondin, E. J. Dickinson, and S. Fortin, "A minimal realization approach to reduced-order modelling and modal analysis for power system response signals," Power Systems, IEEE Transactions on, vol. 8, no. 3, pp. 1020-1029, 1993.

[6] J. J. Sanchez-Gasca and J. H. Chow, "Computation of power system low-order models from time domain simulations using a Hankel matrix," Power Systems, IEEE Transactions on, vol. 12, no. 4, pp. 1461-1467, 1997.

[7] J. J. Sanchez-Gasca and J. H. Chow, "Performance comparison of three identification methods for the analysis of electromechanical oscillations," Power Systems, IEEE Transactions on, vol. 14, no. 3, pp. 995-1002, 1999.

[8] L. Guoping, J. Quintero, and V. Venkatasubramanian, "Oscillation monitoring system based on wide area synchrophasors in power systems," in Proceedings of the iREP Symposium on Bulk Power System Dynamics and Control - VII. Revitalizing Operational Reliability, pp. 1-13, 2007.

[9] J. Hauer, C. Demeure, and L. Scharf, "Initial results in Prony analysis of power system response signals," Power Systems, IEEE Transactions on, vol. 5, no. 1, pp. 80-89, 1990.

[10] R. Kumaresan and D. Tufts, "Estimating the parameters of exponentially damped sinusoids and pole-zero modeling in noise," IEEE Transactions on Acoustics, Speech, and Signal Processing, vol. 30, pp. 833-840, Dec 1982.

[11] R. Kumaresan, D. W. Tufts, and L. L. Scharf, "A Prony method for noisy data: Choosing the signal components and selecting the order in exponential signal models," Proceedings of the IEEE, vol. 72, pp. 230-233, Feb 1984.
[12] D. I. Trudnowski, "Order reduction of large-scale linear oscillatory system models," IEEE Transactions on Power Systems, vol. 9, pp. 451-458, Feb 1994.

[13] N. Zhou, J. W. Pierre, and D. Trudnowski, "A stepwise regression method for estimating dominant electromechanical modes," IEEE Transactions on Power Systems, vol. 27, pp. 1051-1059, May 2012.

[14] S. Zhao and K. A. Loparo, "Forward and backward extended Prony (FBEP) method for power system small-signal stability analysis," IEEE Transactions on Power Systems, vol. 32, pp. 3618-3626, Sept 2017.

[15] R. Kumaresan, "On the zeros of the linear prediction-error filter for deterministic signals," IEEE Transactions on Acoustics, Speech, and Signal Processing, vol. 31, pp. 217-220, Feb 1983.

[16] L. Scharf, Statistical Signal Processing, vol. 98. Addison-Wesley Reading, 1991.

[17] D. J. Trudnowski, J. M. Johnson, and J. F. Hauer, "Making Prony analysis more accurate using multiple signals," Power Systems, IEEE Transactions on, vol. 14, no. 1 , pp. 226-231, 1999.

[18] E. W. Kamen and B. S. Heck, Fundamentals of Signals and Systems Using the Web and MATLAB (3rd Edition). Pearson, 2006.

[19] N. Zhou, Z. Huang, F. Tuffner, J. Pierre, and S. Jin, "Automatic implementation of Prony analysis for electromechanical mode identification from phasor measurements," in Proceedings of the IEEE Power and Energy Society General Meeting, pp. 1-8, 2010.

[20] L. Dosiek, J. W. Pierre, and J. Follum, "A recursive maximum likelihood estimator for the online estimation of electromechanical modes with error bounds," Power Systems, IEEE Transactions on, vol. 28, no. 1, pp. 441-451, 2013.

[21] P. Stoica and R. L. Moses, Spectral Analysis of Signals. Pearson/Prentice Hall Upper Saddle River, NJ, 2005.

[22] R. W. Wies, J. W. Pierre, and D. J. Trudnowski, "Use of ARMA block processing for estimating stationary low-frequency electromechanical modes of power systems," Power Systems, IEEE Transactions on, vol. 18, no. 1, pp. 167-173, 2003.

[23] D. Trudnowski, D. Kosterev, and J. Undrill, "PDCI damping control analysis for the western North American power system," in Power and Energy Society General Meeting (PES), 2013 IEEE, pp. 1-5, July 2013.

[24] S. Kay, Fundamentals of Statistical Signal Processing, Volume I: Estimation Theory. Prentice Hall, 1993. 